\title{
ROR1-CAR T cells are effective against lung and breast cancer in advanced microphysiologic 3D tumor models
}

\author{
Lars Wallstabe, ${ }^{1}$ Claudia Cöttlich,, ${ }^{2,3}$ Lena C. Nelke, ${ }^{2}$ Johanna Kühnemundt, ${ }^{2}$ Thomas Schwarz, ${ }^{2,3}$ \\ Thomas Nerreter, ${ }^{1}$ Hermann Einsele, ${ }^{1}$ Heike Walles, ${ }^{2,3}$ Gudrun Dandekar, ${ }^{2,3}$ Sarah L. Nietzer, ${ }^{2}$ \\ and Michael Hudecek ${ }^{1}$ \\ 'Medizinische Klinik und Poliklinik II and ${ }^{2}$ Tissue Engineering and Regenerative Medicine, Universitätsklinikum \\ Würzburg, Würzburg, Germany. ${ }^{3}$ Fraunhofer Institute for Silicate Research, Translational Center Regenerative \\ Therapies, Würzburg, Germany.
}

Solid tumors impose immunologic and physical barriers to the efficacy of chimeric antigen receptor (CAR) T cell therapy that are not reflected in conventional preclinical testing against singularized tumor cells in 2-dimensional culture. Here, we established microphysiologic three-dimensional (3D) lung and breast cancer models that resemble architectural and phenotypical features of primary tumors and evaluated the antitumor function of receptor tyrosine kinase-like orphan receptor 1specific (ROR1-specific) CAR T cells. 3D tumors were established from A549 (non-small cell lung cancer) and MDA-MB-231 (triple-negative breast cancer) cell lines on a biological scaffold with intact basement membrane (BM) under static and dynamic culture conditions, which resulted in progressively increasing cell mass and invasive growth phenotype (dynamic > static; MDA-MB-231 > A549). Treatment with ROR1-CAR T cells conferred potent antitumor effects. In dynamic culture, CAR T cells actively entered arterial medium flow and adhered to and infiltrated the tumor mass. ROR1-CAR T cells penetrated deep into tumor tissue and eliminated multiple layers of tumor cells located above and below the BM. The microphysiologic 3D tumor models developed in this study are standardized, scalable test systems that can be used either in conjunction with or in lieu of animal testing to interrogate the antitumor function of CAR T cells and to obtain proof of concept for their safety and efficacy before clinical application.

Authorship note: LW, CG, LCN, and JK contributed equally to this work. SLN, GD, and MH contributed equally to this work.

Conflict of interest: $\mathrm{MH}$ is an invento on patents related to chimeric antigen receptor technologies that have been filed by the Fred Hutchinson Cancer Research Center (Seattle, Washington, USA) and the Julius Maximilians University Würzburg (Würzburg, Germany).

Copyright: (c) 2019, American Society for Clinical Investigation.

Submitted: December 4, 2018

Accepted: August 8, 2019

Published: September 19, 2019.

Reference information: JCl Insight. 2019;4(18):e126345.

https://doi.org/10.1172/jci.

insight.126345.

\section{Introduction}

Adoptive immunotherapy with gene-engineered chimeric antigen receptor (CAR) T cells has obtained clinical proof of concept in hematologic cancers with CD19 as an exemplary target antigen in B cell leukemia and lymphoma (1-3). A current focus in the field is to expand applications of CAR T cell therapy from relatively rare hematologic malignancies to prevalent solid tumors. We are pursuing the receptor tyrosine kinase-like orphan receptor 1 (ROR1) as a target antigen for CAR T cells in several hematologic malignancies and solid tumors $(4,5)$. Uniform high-level expression of ROR1 protein was first shown in B cell chronic lymphocytic leukemia, mantle cell lymphoma, and a subset of acute lymphoblastic leukemia and recently also in distinct histologic subsets of prevalent epithelial cancers, including lung, breast, colon, pancreas, renal, and ovarian cancers (6-8). We have demonstrated in previous work that ROR1 has only very limited expression in normal adult tissues and performed toxicology studies in nonhuman primates that did not reveal toxicity of ROR1-directed immunotherapy to vital normal organs (9). In addition, there is evidence for a critical role of ROR1 in malignant transformation and oncogenic signaling that supports the use of ROR1 as a target in cancer immunotherapy (10).

We have recently reported on the development of a ROR1-specific CAR with optimized targeting domain affinity and extracellular spacer design that confers specific recognition and potent reactivity against ROR $1^{+}$ lymphoma in preclinical models in vitro and in vivo $(5,11)$. We have also demonstrated that $\mathrm{CD}^{+} \mathrm{T}$ cells expressing this ROR1-CAR are capable of conferring cytolysis of MDA-MB-231 triple-negative breast cancer (TNBC) cells in conventional 2D coculture (5). Although encouraging, the use of singularized tumor cells in $2 \mathrm{D}$ culture is simplistic and does not consider the specific challenges in tumor architecture, phenotype, and 
microenvironment that CAR T cells encounter when combating a solid tumor. In the present study we are therefore evaluating the antitumor function of ROR1-CAR T cells in microphysiologic 3D tumor models that reflect these challenges more realistically and provide an alternative to testing in animal models.

We have in previous work established protocols for the engineering of vascularized and nonvascularized 3D tissues in bioreactors (BioVaSc-TERM technology) (12). The BioVaSc is derived from decellularized porcine jejunum and used as a collagen scaffold with intact basement membrane (BM) that permits the engraftment and propagation of normal and cancerous epithelial tissues in 3D culture. We have recently demonstrated the development of a 3D lung tumor from A549 non-small cell lung cancer (NSCLC) using the BioVaSc technology (13-15). The A549 tumor model uses the nonvascular part of the BioVaSc - the small intestinal submucosa and mucosa (SISmuc) - for cell engraftment. Intriguingly, the resulting lung tumor resembled the architecture of primary lung carcinomas and recapitulated "real-life" pathophysiologic features, including growth kinetic, tumor cell migration and invasion, and epithelial-mesenchymal transition after stimulation with TGF- $\beta 1(13,14)$.

Here we established microphysiologic 3D tumor models of A549 NSCLC and MDA-MB-231 TNBC using the SISmuc technology to assess the antitumor function of ROR1-CAR T cells. We obtained 3D tumors with considerable cell mass and demonstrate that ROR1-CAR T cells rapidly infiltrate and migrate through the 3D tumor tissue and confer a specific and potent antitumor effect over a several-day time course. ROR1CAR T cells were able to sustain their cytolytic activity, cytokine secretion, and proliferation for several days in culture but displayed functional and phenotypic indicators of $\mathrm{T}$ cell exhaustion at the end of the analysis period. The data illustrate the utility of microphysiologic 3D culture systems for evaluating CAR T cell performance beyond the scope of conventional testing in $2 \mathrm{D}$ in vitro and in xenograft animal models.

\section{Results}

Invasive growth of A549 lung and $M D A-M B-231$ breast cancer in 3D culture. We established microphysiologic 3D models of NSCLC (A549 cell line) and TNBC (MDA-MB-231 cell line) using the SISmuc platform. First, we applied static culture conditions in cell crowns (i.e., 2 metal rings for matrix fixation) and performed histopathologic analysis on cross sections of the resulting tumors after 14 days of incubation (Figure 1 and Supplemental Figure 1A; supplemental material available online with this article; https://doi.org/10.1172/jci.insight.126345DS1). Staining was performed for the epithelial marker pan-cytokeratin (PCK) to detect tumor cells and collagen IV (Col IV) to detect extracellular matrix (ECM). Tumor architecture was evaluated using a customized grading system (Table 1). We found that A549 tumor cells formed a confluent monolayer that populated the entire SISmuc area and settled deep into crypts. However, A549 cells did not cross the BM (grade 1) (Figure 1). The distribution of MDA-MB-231 cells was more scattered on the SISmuc surface and denser within crypts. Remarkably, there were MDA-MB-231 cells that had crossed the BM barrier and had migrated into the matrix of the former mucosa compartment (grade 2) (Figure 1). For each cell line, this architecture and phenotype was confirmed in more than 10 replicate experiments that we established on distinct batches of SISmuc scaffold.

In the next set of experiments, we applied dynamic culture conditions in a flow bioreactor to establish a larger tumor mass of A549 and MDA-MB-231 cells on the SISmuc platform. Histopathologic analysis was performed on day 14 after transfer to the bioreactor (Figure 1 and Supplemental Figure 1B). We found that the mass of A549 cells had considerably increased compared with the static culture model and comprised several layers of tumor cells that spanned the entire scaffold surface. In addition, there was a higher degree of migration, with A549 cells packing the entire lumen of crypt structures and invasion of A549 cells across the BM (grade 2) (Figure 1). Also with MDA-MB-231 cells, there was a substantial increase in tumor mass in the dynamic compared with the static culture system and a shift to an aggressive and destructive growth phenotype. The data show that MDA-MB-231 cells infiltrated the entire SISmuc matrix and crossed the barrier between former mucosa and submucosa compartments (grade 3). Accordingly, the MDA-MB-231 tumors displayed high-level expression of the mesenchymal marker vimentin, consistent with epithelial-mesenchymal transition (Figure 1). This architecture and phenotype were obtained consistently in more than 10 independent experiments with distinct batches of SISmuc scaffold. We confirmed that A549 and MDA-MB-231 cells that we isolated from the 3D tumors had retained uniform expression of the ROR1 antigen (Supplemental Figure 2).

Taken together, these data show that microphysiologic 3D tumor models of breast and lung cancer can be established on the SISmuc platform using the A549 and MDA-MB-231 cell lines. The use of static and dynamic culture conditions, respectively, provides tumors with increasing cell mass and distinct architectural features that permit interrogating the antitumor function of CAR T cells in an increasingly challenging microenvironment. 


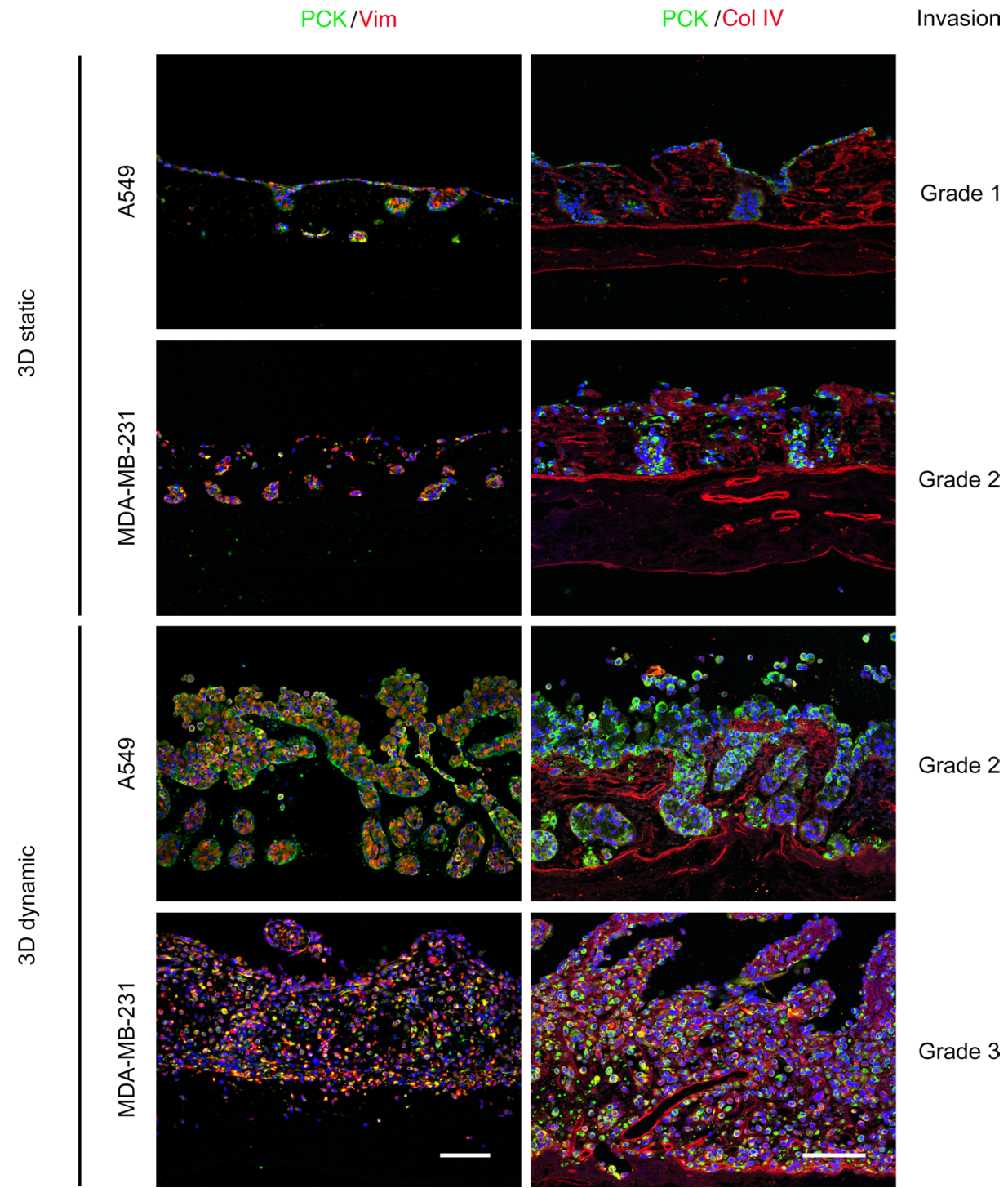

Figure 1. Invasive growth of A549 lung cancer and MDA-MB-231 breast cancer in 3D culture. The tumor cell lines A549 (lung cancer) and MDA-MB-231 (breast cancer) were cultured on SISmuc scaffolds under static and dynamic culture conditions. Tumor composition and architecture were evaluated by immunofluorescence staining Left column: pan-cytokeratin (PCK, shown in green) and vimentin (Vim, shown in red). Nuclei are counterstained with DAPI (blue). Right column: PCK (green) and collagen IV (Col IV, red). Nuclei are counterstained with DAPI (blue). Scale bars: $100 \mu \mathrm{m}$ (lower images, representative for all images of a column). Grading was performed according to the scheme presented in Table 1.

ROR1-CAR T cells eliminate large $3 D$ masses of lung and breast cancer. We sought to determine whether ROR1-specific CAR T cells are able to mediate an antitumor effect in the 3D A549 lung and MDA-MB-231 breast cancer models under static conditions (grades 1 and 2, respectively). In this set of experiments, we used an optimized ROR1-CAR comprising a targeting domain specific for the R12 epitope, a short IgG4-Fc

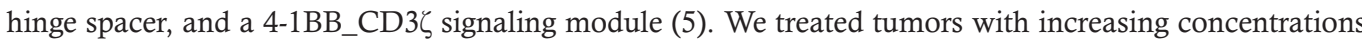
of CD8 $8^{+}$ROR1-CAR-modified or non-CAR-modified control T cells (Figure 2A) (dose range: $5 \times 10^{4}$ to 1 $\times 10^{6}$ ) and performed M30 ELISA to detect caspase-cleaved keratin 18 as a measure of tumor cell apoptosis over a 3-day period. Experiments were done in $n=4$ biological replicates with $\mathrm{T}$ cell lines from different donors. Indeed, we found that ROR1-CAR T cells conferred a specific and potent antitumor effect in the 3D models. There was a higher M30 ELISA signal at each analysis time point (starting at 24 hours) and in each of the A549 and MDA-MB-231 cell crowns that we had treated with ROR1-CAR T cells compared with control T cells (Figure 2B). The antitumor effect was dose dependent; e.g., with higher doses of CAR $\mathrm{T}$ cells, there was a higher peak ELISA signal, and the peak signal occurred earlier during the 3-day analysis 
Table 1. Grades of invasiveness represented by the 3D tumor models

\begin{tabular}{lcc}
\hline Grade & Phenotype & Represented by model \\
1 & Tumor cells populate former crypt structures but do not cross the basement membrane & A549, static culture \\
2 & Single tumor cells cross the BM; crypt structure is preserved & MDA-MB-231, static culture/ A549, dynamic culture \\
3 & Tumor cells cross the BM and infiltrate the extracellular matrix (ECM), thereby & MDA-MB-231, dynamic culture \\
& disrupting crypt structure morphology
\end{tabular}

period. When we administered $5 \times 10^{5}$ or $1 \times 10^{6}$ ROR1-CAR T cells to A549 lung cancer, the peak ELISA signal was obtained during the 6- to 24-hour time interval, with an 11.2-fold and 14.5-fold increase in apoptosis induction compared with control T cells, respectively (Figure 2B). When we administered $2.5 \times$ $10^{5}$ ROR1-CAR T cells to A549 lung cancer, the peak ELISA signal was obtained during the 24- to 48-hour interval, and at doses lower than $2.5 \times 10^{5}$ ROR1-CAR T cells, the peak signal was obtained during the 48- to 72-hour interval (Figure 2B). Of note, the M30 ELISA specifically measures apoptosis of cells with epithelial phenotype, and accordingly, we obtained a higher overall signal in the A549 lung cancer model compared with the MDA-MB-231 breast cancer model because the 3D tumors derived from MDA-MB-231 displayed a partly mesenchymal phenotype (Figure 1).

We obtained medium samples at 6 hours, 24 hours, 48 hours, and 72 hours during the 3-day assay period and detected high levels of IFN- $\gamma$ at each analysis time point after treatment with ROR1-CAR T cells. The amount of IL-2 that we detected by ELISA declined between the 24-hour and 72-hour time points, indicating that IL-2 had been consumed by activated CAR T cells (Figure 2C). The magnitude of IFN- $\gamma$ and IL-2 release by ROR1-CAR T cells was similar in the A549 and the MDA-MB-231 models. Flow cytometric analysis on $\mathrm{T}$ cells on day 3 showed uniform expression of the activation markers CD25 and CD69 on ROR1-CAR T cells but not on non-CAR-modified control T cells (Figure 2D). Together, these data show that ROR1-CAR T cells mediate a specific antitumor effect against 3D microphysiologic tumor models of A549 lung cancer and MDA-MB-231 breast cancer under static culture conditions.

ROR1-CAR T cells sustain their antitumor function for several days in 3D culture. We extracted the SISmuc scaffolds on day 3 after $\mathrm{T}$ cell administration and prepared cross sections for immunofluorescent staining. We performed costaining for CD45 and DAPI to distinguish T cells $\left(\mathrm{CD} 45^{+} \mathrm{DAPI}{ }^{+}\right.$) and tumor cells $\left(\mathrm{CD} 45^{-} \mathrm{DAPI}^{+}\right)$. These analyses confirmed that ROR1-CAR T cells had conferred a specific and potent antitumor effect in the A549 NSCLC and MDA-MB-231 TNBC models. In particular, the data show that ROR1-CAR T cells had attached to the apical tumor cell layer, had migrated through the BM and SISmuc matrix, and could be detected in the former mucosa compartment (Figure 3A). On high-power resolution, the direct interaction of ROR1-CAR T cells with A549 and MDA-MB-231 tumor cells could be visualized (Figure 3B). Overall, the number of infiltrating $\mathrm{T}$ cells in the scaffold correlated with the number of $\mathrm{T}$ cells that had been administered (Figure 3A). Even at the lowest CAR T cell dose level, a substantial proportion of tumor cells had been eliminated, regardless of how deeply tumor cells had migrated into crypts and the tissue matrix. Costaining for CD45 and Ki67 and cell counting confirmed that there was an increase in absolute ROR1-CAR T cell number on day 3 after administration and a high percentage of proliferating ROR1-CAR T cells (Figure 3C).

In summary, these data show that ROR1-CAR $\mathrm{T}$ cells are able to infiltrate and migrate through the tumor matrix and maintain their function for at least 72 hours after administration under static culture conditions. ROR1-CAR T cells were capable of eliminating tumor cells that were harbored in crypt structures and ECM on the contralateral side of the BM, indicating they possess and maintain a substantial migratory capacity within the tumor mass.

Programmed cell death 1 induction on CAR T cells after exposure to tumor cells in $3 D$ culture. We analyzed expression of programmed cell death 1 (PD-1) on ROR1-CAR-modified and non-CAR-modified control T cells at the end of the assay period in the static A549 lung cancer and MDA-MB-231 breast cancer models. We detected PD-1 on both $\mathrm{CD}^{+}$and CD4 ${ }^{+}$ROR1-CAR T cells (CD4 > CD8) (Supplemental Figure 3, A and B). There were higher levels of PD-1 on ROR1-CAR T cells that had been exposed to MDA-MB-231 breast cancer cells compared with A549 lung cancer cells (Supplemental Figure 3, A and B). ROR1-CAR T cells that we had maintained in assay medium without exposure to tumor cells served as reference. No significant difference in PD-1 expression was detected between control $\mathrm{T}$ cells that had been exposed to tumor cells or 
A

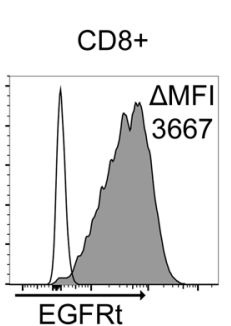

B

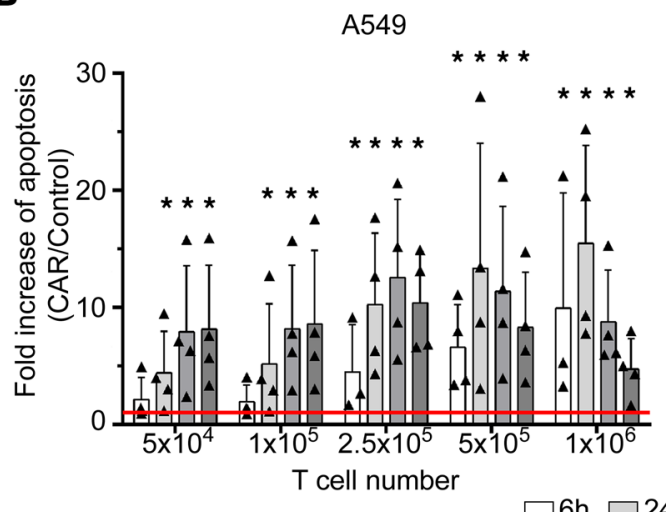

D $\quad$ A549

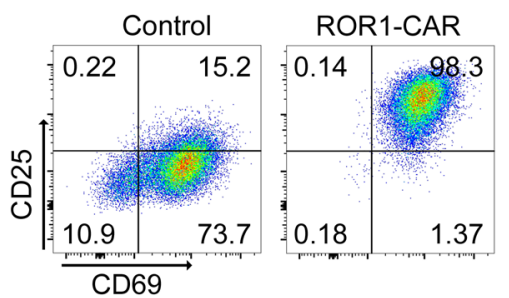

MDA-MB-231

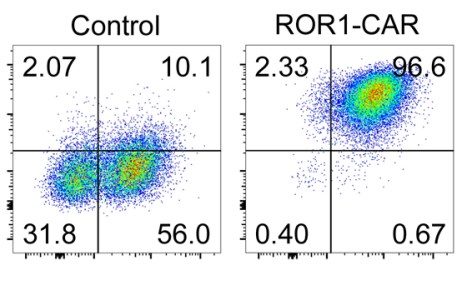

C

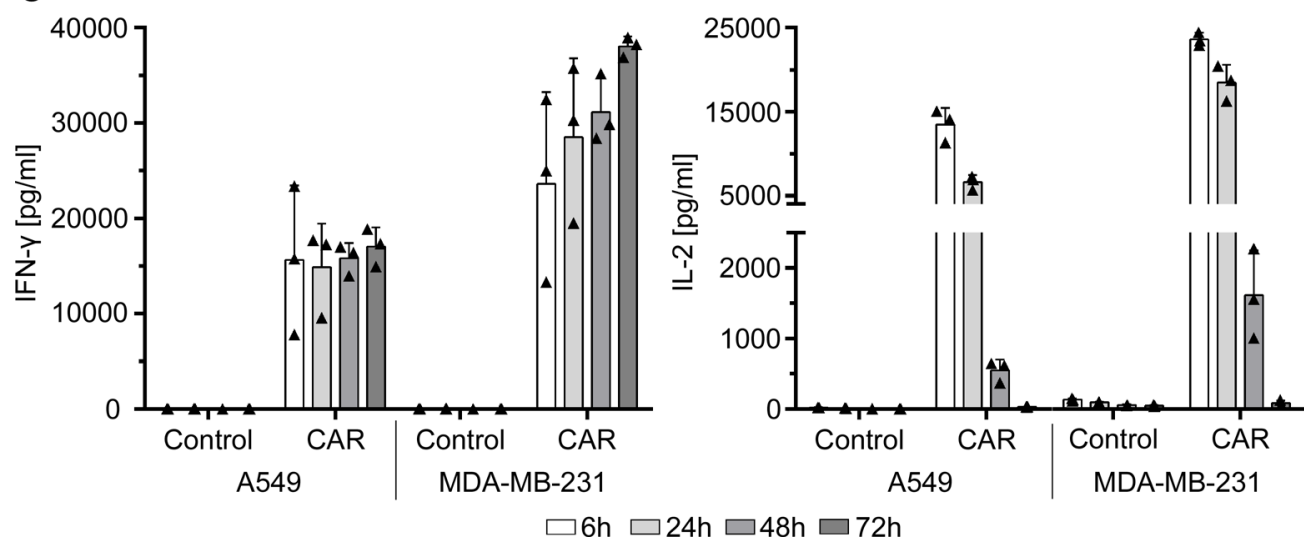

Figure 2. ROR1-CAR T cells induce apoptosis of $3 D$ lung cancer and breast cancer in static culture. (A) Expression of truncated epidermal growth factor receptor (EGFRt) transduction marker on CD8 ${ }^{+}$ROR1-CAR T cells before functional testing. $\triangle \mathrm{MFI}$ depicts the difference in geometric mean fluorescence intensity between ROR1-CAR T cells and unmodified control T cells. (B) Quantification of apoptosis induced by ROR1-CAR T cell treatment with increasing CD8 ${ }^{+} T$ cell numbers for 72 hours. Apoptosis was measured with M30 ELISA from supernatants collected at the indicated time points and is presented as fold change compared with the respective control T cell treatment (red line). $n=4$. Data are presented as arithmetic mean \pm SD, Wilcoxon's rank-sum test: ${ }^{*} P<0.05$. (C) ELISA-based quantification of IFN- $\gamma$ and IL-2 from supernatants collected at the indicated time points from static tumor models treated with $1 \times 10^{6} \mathrm{~T}$ cells for 72 hours. Data are presented as arithmetic mean of 3 cell crowns \pm SD. $n=1$ experiment. (D) Expression of CD25 and CD69 on CD8 ${ }^{+}$ROR1-CAR T cells and unmodified control T cells at the end of the 72-hour analysis period in the static tumor model. One representative plot of $n=3$ experiments is shown.

maintained in assay medium. Of note, we detected uniform expression of programmed cell death ligand 1 (PD-L1) on MDA-MB-231 cells and A549 cells (MDA-MB-231 > A549) (Supplemental Figure 3C). These data confirm that ROR1-CAR T cells undergo specific activation in 3D culture and suggest that, depending on the particular tumor model, CAR T cells enter differential grades of activation and exhaustion.

Variations in CAR targeting domain and spacer design affect antitumor function in $3 D$ culture. We were interested in determining whether testing in 3D culture can disclose differences in antitumor function between ROR1CAR constructs that comprise distinct targeting domains and spacer designs. We used the ROR1-CAR with

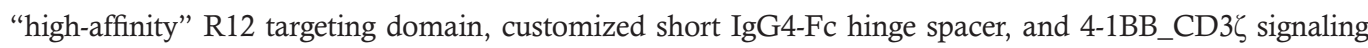
module that we had previously "optimized" in $2 \mathrm{D}$ in vitro culture and in xenograft in vivo models as refer- 
A

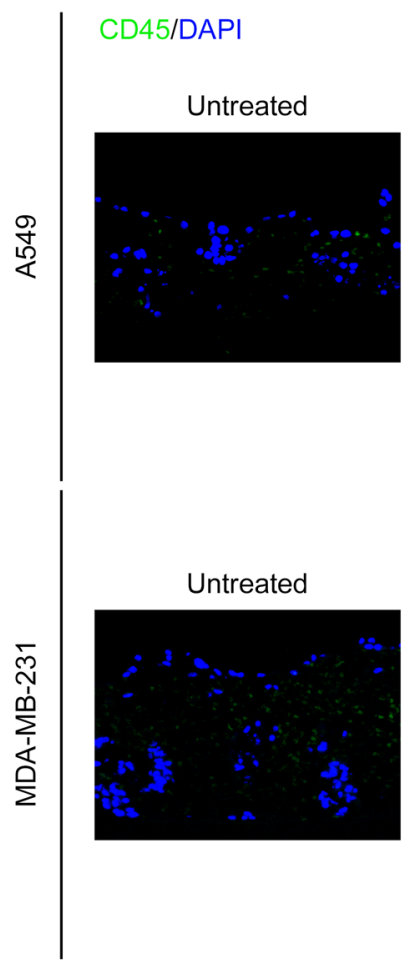

B

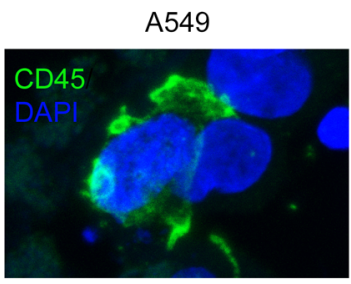

$5 \times 10^{4}$
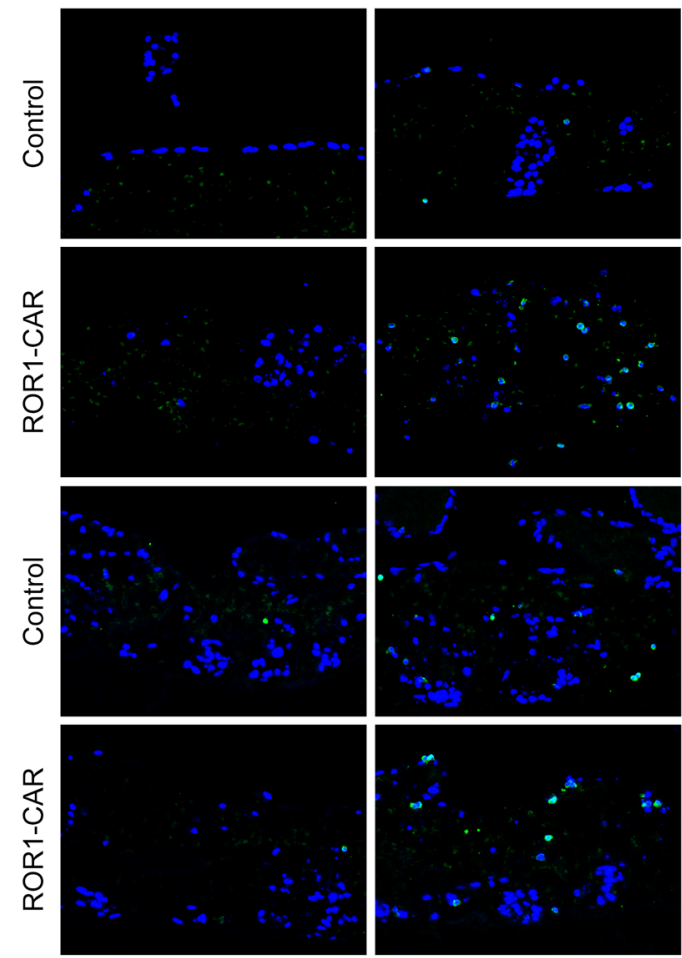

C
$2.5 \times 10^{5}$
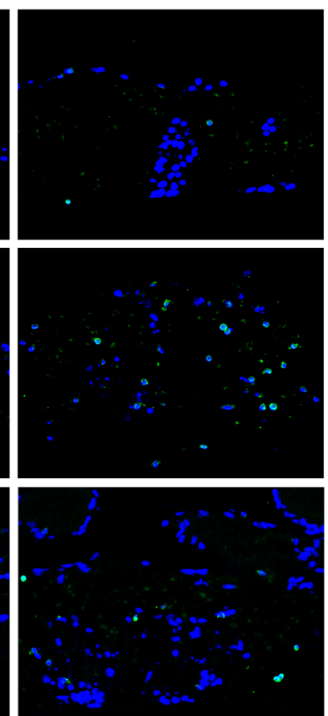

MDA-MB-231

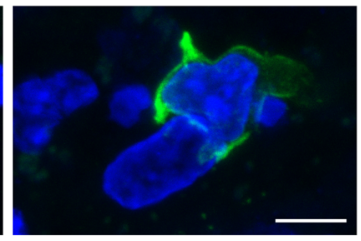

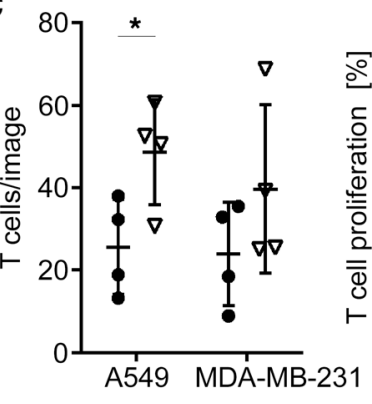

- Control $\nabla$ ROR1-CAR
$1 \times 10^{6}$
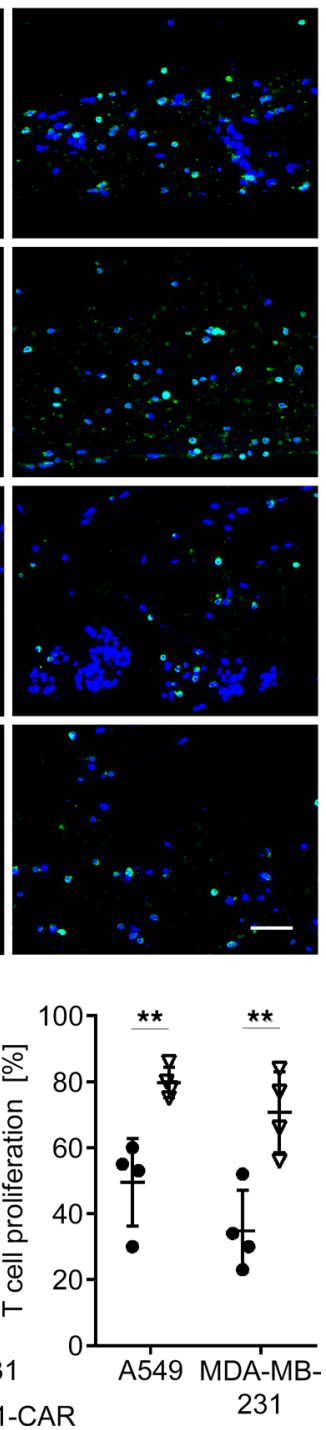

Figure 3. ROR1-CAR T cells migrate into tumor tissue and proliferate in static 3D culture. (A) Immunofluorescence staining of lymphocyte marker CD45 (green) on paraffin sections of untreated tumor models (Untreated) or tumor models treated with increasing doses $\left(5 \times 10^{4}, 2.5 \times 10^{5}, 1 \times 10^{6}\right.$ cells) of unmodified CD8 $8^{+}$control T cells (Control) or CD8 ${ }^{+}$ROR1-CAR T cells (ROR1-CAR). Nuclei are counterstained with DAPI (blue). Scale bar: $50 \mu \mathrm{m}$. (B) Close-up of CD45 immunofluorescence staining (green) on paraffin sections of CD8 ${ }^{+}$ROR1-CAR T cell-treated 3D tumor models. Nuclei are counterstained with DAPI (blue). Scale bar: $10 \mu \mathrm{m}$. (C) Mean number of T cells per image and proliferation of T cells in static 3D tumor models treated with $5 \times 10^{4}$ unmodified CD $8^{+}$control T cells (Control) or CD8 ${ }^{+}$ROR1-CAR T cells (ROR1-CAR) assessed by quantification of Ki67/CD45 immunofluorescence double staining. CD45 ${ }^{+}$and Ki67+CD45 ${ }^{+}$ cells were counted in 10 images per condition. $n=4$. Data are presented as arithmetic mean \pm SD, 2-tailed Student's $t$ test: ${ }^{*} P<0.05$; ${ }^{* *} P<0.01$.

ence and used a "suboptimal" ROR1-CAR with "low-affinity" 2A2 targeting domain for comparison (5). In this set of experiments, we used the A549 lung cancer model under static culture conditions. First, we showed that the 2A2 ROR1-CAR worked best with a short IgG4-Fc hinge spacer rather than a long IgG4Fc hinge- $\mathrm{C}_{\mathrm{H}} 2-\mathrm{C}_{\mathrm{H}} 3$ spacer domain, as demonstrated by superior production of IFN- $\gamma$ and IL-2 in 3D culture (Supplemental Figure 4). This confirmed our prior data in conventional 2D in vitro culture that had identified the short spacer as the optimal configuration for targeting the 2A2 ROR1 epitope (5). Then, we compared the antitumor function of $\mathrm{T}$ cells expressing the R12 and 2A2 ROR1-CAR constructs in 3D culture. We found stronger apoptosis induction with the R12 ROR1-CAR, most evident when ROR1-CAR T cells were administered at a low dose $\left(5 \times 10^{4}\right)$. At this dose, the peak signal in the M30 ELISA was obtained in the 24- to 48-hour interval. When ROR1-CAR T cells were administered at higher doses $\left(2.5 \times 10^{5}\right.$ to $\left.1 \times 10^{6}\right)$, the M30 ELISA signal obtained with the 2 ROR1-CAR constructs was similar. At the $1 \times 10^{6}$ dose level, the peak M30 
ELISA signal was already obtained in the 6- to 24-hour interval (Figure 4A). The R12 ROR1-CAR induced stronger IFN- $\gamma$ and IL-2 production compared with the 2A2 ROR1-CAR at each of the analysis time points. Peak IFN- $\gamma$ production occurred in the 6- to 24 -hour interval, and peak IL-2 production occurred in the first 6 hours of the assay period (Figure 4B). By flow cytometric analysis, we detected uniform expression of the activation markers CD25 and CD69 on R12 ROR1-CAR T cells and 2A2 ROR1-CAR T cells at the end of the 72-hour assay period (Figure 4C). From this comparison, the R12 ROR1-CAR emerged as the superior construct and was therefore advanced to further evaluation in dynamic $3 \mathrm{D}$ culture.

Taken together, these data show that ROR1-CAR constructs that differ in targeting domain and spacer design confer different degrees of reactivity in $3 \mathrm{D}$ culture. These data affirm our prior notion that variations in CAR design affect tumor recognition and CAR $\mathrm{T}$ cell function and suggest that test campaigns in $3 \mathrm{D}$ tumor models can be performed to identify CAR designs with optimal and suboptimal reactivity.

ROR1-CAR T cells penetrate and destroy invasive 3D tumors in dynamic culture. Next, we assessed the performance of R12 ROR1-CAR T cells against A549 lung cancer and MDA-MB-231 breast cancer in the dynamic culture system with constant medium flow in a closed-circuit setup (grades 2 and 3, respectively). This setup provides additional challenges because $\mathrm{T}$ cells are administered into a medium reservoir and have to (a) enter arterial medium flow, (b) adhere to and penetrate into the SISmuc tumor matrix, and (c) encounter a larger number of tumor cells compared with the static model. We treated tumors with a fixed dose of $10 \times 10^{6}$ ROR1-CAR T cells (CD8 ${ }^{+} / \mathrm{CD}^{+}$at 1:1 ratio) (16) and performed M30 ELISA at 24-hour intervals over a 5-day period. Experiments were done in $n=4$ biological replicates with $\mathrm{T}$ cell lines from different donors. We detected specific and very potent antitumor activity of ROR1-CAR T cells against A549 lung cancer and MDA-MB-231 breast cancer (Figure 5A). With A549 lung cancer, apoptosis induction peaked between 24 hours and 48 hours after ROR1-CAR T cell transfer (7.7-fold higher compared with control T cells) and continued over the entire 5-day period (Figure 5A). With MDA-MB-231 breast cancer, apoptosis induction peaked in the first 24 hours after CAR T cell administration (3.6-fold higher compared with control T cells) and subsequently declined. Analysis of the culture medium by ELISA revealed that CAR T cells had produced very high levels of IFN- $\gamma$ and IL-2 (Figure 5B). At the end of the 5-day assay period, $\mathrm{CD}^{+}$and $\mathrm{CD} 4{ }^{+}$ROR1-CAR $\mathrm{T}$ cells displayed an activated phenotype as assessed by expression of CD25 and CD69 (Figure 5C and Supplemental Figure 5).

On day 5, we performed a detailed histopathologic analysis on the SISmuc scaffolds. We found that ROR1-CAR T cells $\left(\mathrm{CD}^{2} 5^{+} \mathrm{DAPI}{ }^{+}\right)$had migrated throughout the entire tumor matrix (Figure 6A, white arrows) and were present at a higher frequency compared with control T cells. In the A549 lung cancer model, ROR1-CAR T cells eliminated multiple layers of tumor cells that had been located on top of the matrix and markedly reduced the number of tumor cells in crypts. Non-CAR-modified control T cells did not convey a discernable antitumor effect. In the MDA-MB-231 breast cancer model, ROR1-CAR T cells had removed a substantial proportion of tumor cells in the entire tissue matrix as evidenced by immune fluorescence staining for CD45/DAPI and PCK/Col IV (Figure 6, A and B). In both tumor models, there was a high percentage of proliferating ROR1-CAR T cells as assessed by Ki67 staining (Figure 6C) and a significant increase in absolute numbers of ROR1-CAR T cells compared with control T cells (Figure 6C).

Collectively, the data show that ROR1-specific CAR T cells confer substantial antitumor reactivity against invasive A549 lung cancer and MDA-MB-231 breast cancer that have grown to comprise large cell masses and display a destructive growth phenotype. ROR1-CAR T cells remain viable for several days in dynamic 3D culture and surmount physical and immunologic barriers to exert their antitumor functions.

\section{Discussion}

The clinical proof of concept for the safety and efficacy of adoptive immunotherapy with CAR T cells in prevalent solid tumors is still pending. The challenges that have been postulated for CAR T cells to overcome include migrating through endothelial and stroma barriers surrounding the tumor cells, withstanding "chronic" antigen exposure in a large tumor mass, and maintaining viability and function in the tumor microenvironment that is poised with immunosuppressive cytokines, cellular ligands, and metabolites (17). Therefore, significant efforts are being invested in preclinical studies that seek to interrogate CAR $\mathrm{T}$ cell antitumor function and to guide the evolution of CAR technologies beyond the point that is deemed necessary for achieving therapeutic efficacy in the clinical setting. A problem is that conventional preclinical test models do not reflect the unique attributes and challenges that solid tumors impose. In the majority of preclinical studies, the potency of CAR T cells is probed against singularized cells of established solid tumor 
A
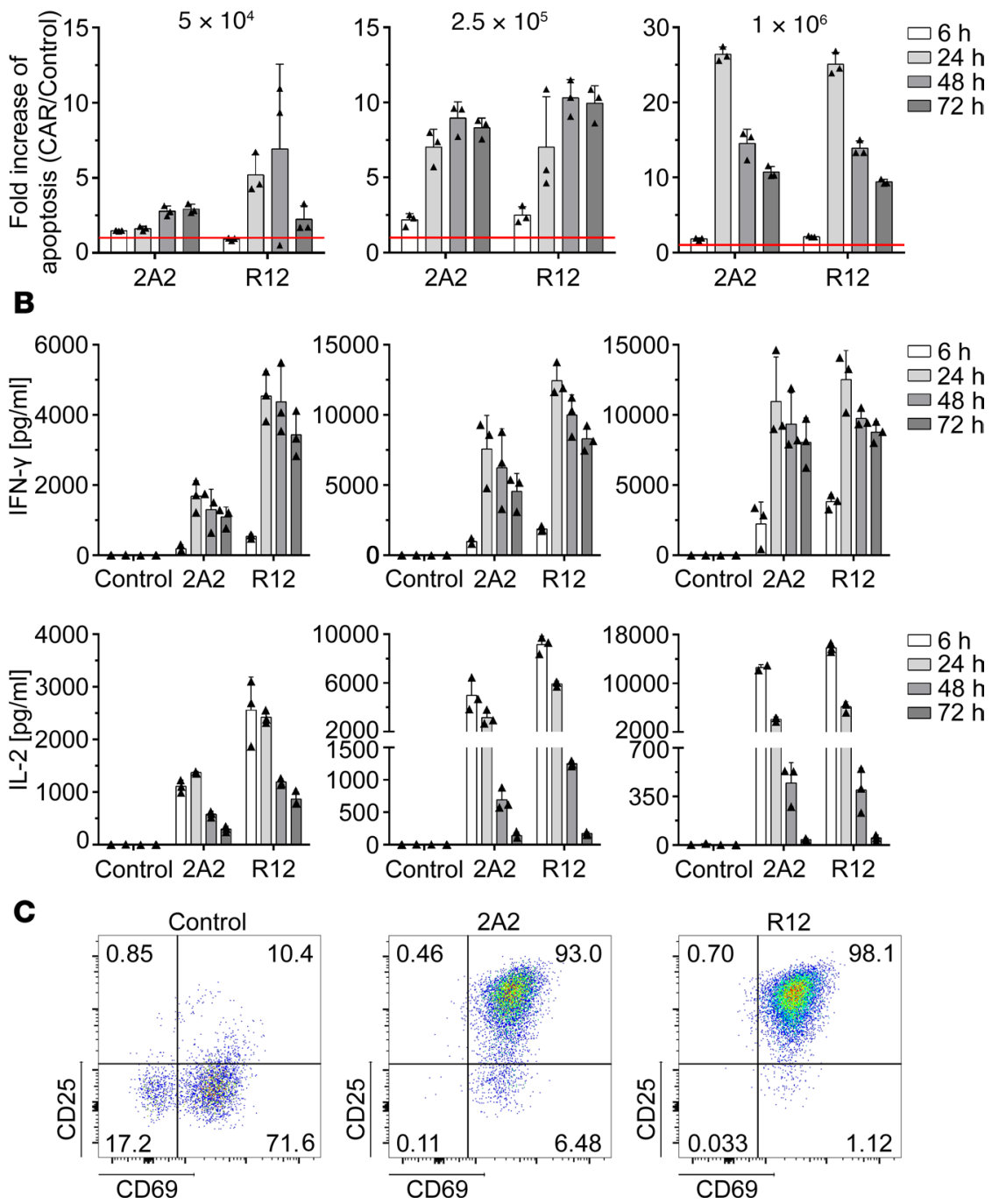

Figure 4. Variations in ROR1-CAR targeting domain affect antitumor function in 3D lung cancer models. (A) Quantification of apoptosis induced by ROR1-CAR T cells with either 2A2 or R12 targeting domains during 72-hour treatment with increasing CD8 ${ }^{+} \mathrm{T}$ cell dose $\left(5 \times 10^{4}, 2.5 \times 10^{5}, 1 \times 10^{6}\right)$. Apoptosis was measured with M30 ELISA from supernatants collected at the indicated time points and is presented as fold change compared with the same dose of control T cells (red line). Data are presented as arithmetic mean of 3 cell crowns \pm SD. $n=1$ experiment. (B) ELISA-based quantification of IFN- $\gamma$ and IL-2 from supernatants collected at the indicated time points from static tumor models treated with increasing CD8 T cell numbers for 72 hours. Data are presented as arithmetic mean of 3 cell crowns \pm SD. $n=1$ experiment. (C) Expression of CD25 and CD69 on CD8 ${ }^{+}$ROR1-CAR T cells and unmodified control T cells at the end of the 72-hour analysis period in the static tumor model. One representative plot of 3 cell crowns from $n=1$ experiment is shown.

cell lines in vitro at effector/target cell ratios that cannot be achieved in humans, and in murine xenograft models, where tumor cell lines are ectopically inoculated and form tumor lesions that do not resemble the architecture and phenotype of primary human tumors (18). Therefore, we set out to develop an alternative test system that incorporates physical and immunologic barriers of solid tumors more realistically and focused on lung and breast cancer as the 2 most prevalent cancers in men and women.

The microphysiologic 3D tumor models used in this study are based on a biological matrix derived from decellularized porcine jejunum that contains an intact $\mathrm{BM}$ and therefore permits rapid engraftment of epithelial cancer cells. We inoculated this matrix with 2 established and widely accepted tumor cell lines, A549 (NSCLC) and MDA-MB-231 (TNBC). Upon static and dynamic culture, tumors could be routinely established and displayed a consistent phenotype with characteristic architecture. There are several lines of evidence supporting the notion that the density and culture environment of immortalized tumor cell lines affect their differentiation, signal transduction, and drug sensitivity $(19,20)$. Indeed, we have shown 

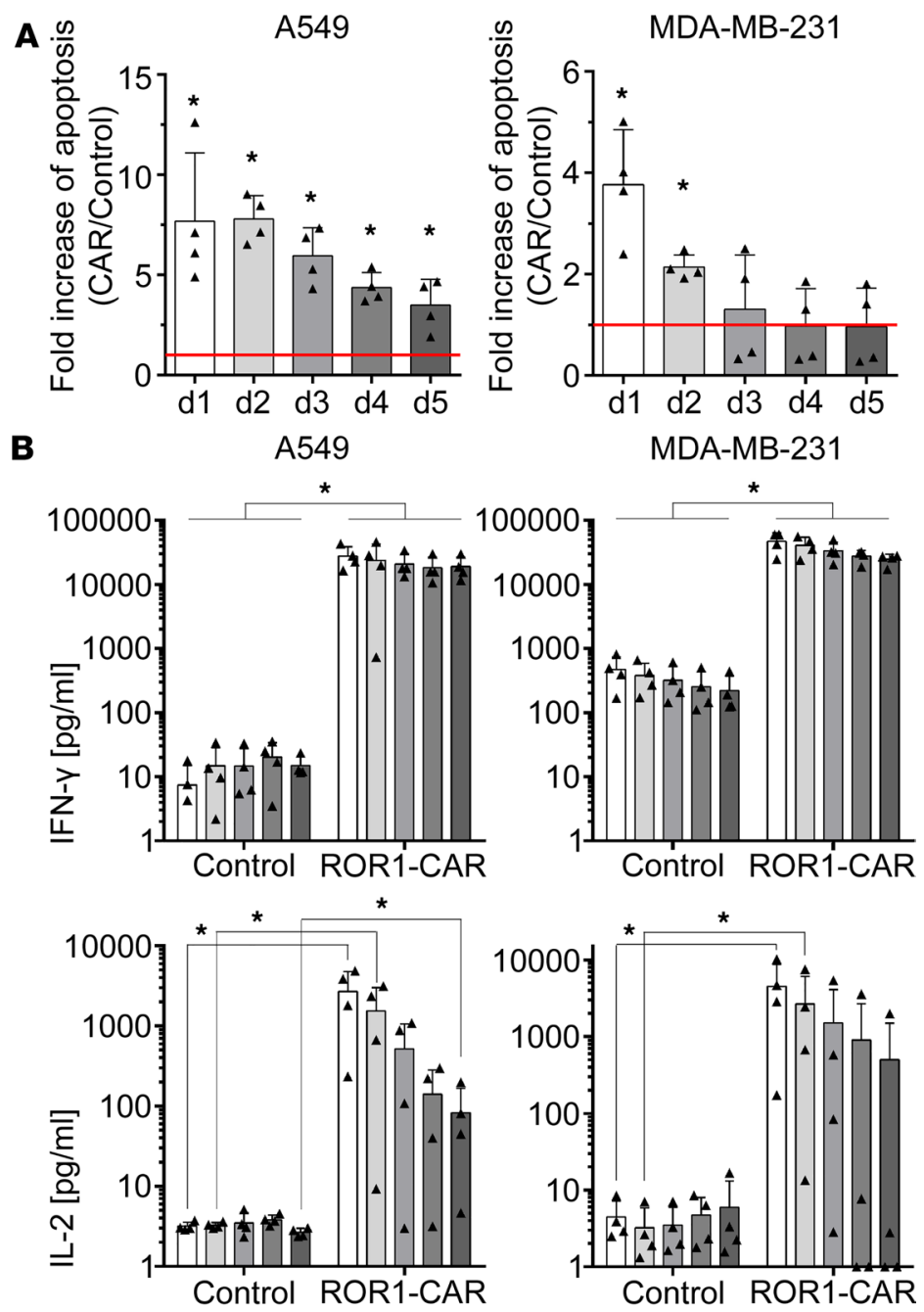

$\square \mathrm{d} 1 \square \mathrm{d} 2 \square \mathrm{d} 3 \square \mathrm{d} 4 \square \mathrm{d} 5$
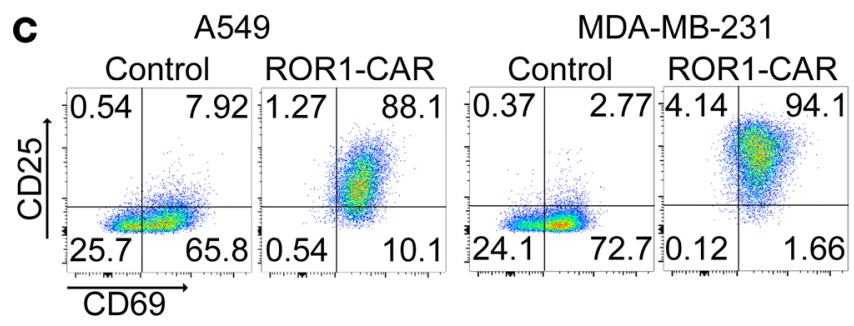

Figure 5. ROR1-CAR T cells induce tumor cell apoptosis of 3D lung and breast cancer in dynamic culture. (A) Quantification of apoptosis induced by ROR1-CAR T cells during 5 days of treatment. Apoptosis was measured with M30 ELISA from supernatants collected at the indicated time points and is presented as fold change compared with the respective control T cell treatment (red line). $n=4$. Data are presented as arithmetic mean $\pm \mathrm{SD}$, Wilcoxon's rank-sum test: ${ }^{*} P<$ 0.05. (B) ELISA-based quantification of IFN- $\gamma$ and IL-2 from supernatants collected over time from dynamic tumor models treated with T cells for 5 days. $n=4$. Data are presented as arithmetic mean $\pm S D$, Wilcoxon's rank-sum test: ${ }^{*} P<0.05$. (C) After 5 days of treatment, $\mathrm{CD}^{+} \mathrm{T}$ cells were analyzed for expression of CD25 and CD69 by flow cytometry. One representative plot of $n=4$ experiments is shown.

in previous studies that tumor cells established on our 3D scaffold have a more realistic proliferation rate and differentiation state, drug sensitivity, and response compared with tumor cells in conventional 2D culture $(13,15)$. In our dynamic $3 \mathrm{D}$ culture models with A549 cells and MDA-MB-231 cells, there were multiple layers of tumor cells and tumor cell aggregates in former crypts that provided a substantially greater challenge to CAR $T$ cells for conferring their antitumor reactivity compared with singularized tumor cells in $2 \mathrm{D}$ culture. We have shown in previous work with normal and malignant tissue models that continuous medium flow in the dynamic 3D culture system results in superior nutrient supply and waste product removal compared with the static culture system (21). Accordingly, the dynamic culture approach yielded a greater tumor cell mass and supported the invasion of tumor cells beyond the BM for both A549 lung cancer and MDA-MB-231 breast cancer in our microphysiologic 3D models. In particular, MDA-MB-231 cells displayed more rapid and more aggressive growth compared with A549 cells, consistent with previous studies in 2D in vitro and organoid culture that described the invasive potential of this cell line (22).

Several strategies for ex vivo testing of tumor-reactive $\mathrm{T}$ cells have been proposed, including the use of suspension cultures or gel cultures with tumor organoids. Tumor organoids have been derived from A549 lung cancer and MDA-MB-231 breast cancer cells $(23,24)$. An advantage of organoids is that they are faster and easier to produce compared with microphysiologic $3 \mathrm{D}$ models and are easier to scale up for high-throughput testing. However, organoids do not resemble primary tumor architecture, and the tumor cell mass is much lower compared with our 3D models. Therefore, several critical features of CAR T cells, including their ability to penetrate and migrate through tumor tissue and their response to chronic antigen exposure, cannot be adequately addressed in organoids. Another approach that has been proposed for evaluating the interaction of $\mathrm{T}$ cells and solid tumor cells is the use of a microscale fluidic device where tumor cells are inserted as single cells or cell aggregates in collagen gel and $\mathrm{T}$ cells are supplemented in a parallel channel with directional medium flow (25). An advantage of this microfluidic model is that it is easier to obtain information on the performance of individual $\mathrm{T}$ cells, even though the spatiotemporal resolution in this setup is low. The microphysiologic $3 \mathrm{D}$ models described in our study are more laborious to establish compared with organoids and more difficult to set up compared with the microfluidic system. Importantly, however, they can be scaled up to medium- and high-throughput testing and provide a global view on CAR T cell performance. This view can even be refined by disassembling the $3 \mathrm{D}$ scaffold at the end of the assay to perform detailed histologic and phenotypic analyses on $\mathrm{T}$ cells, as well as residual tumor and stroma cells. 
A
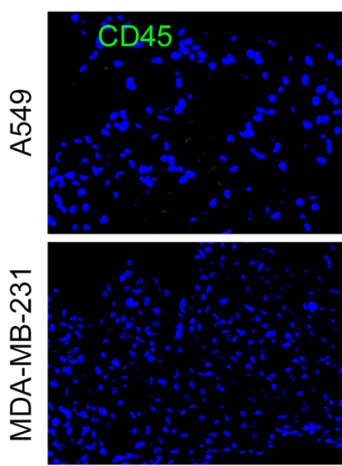

B
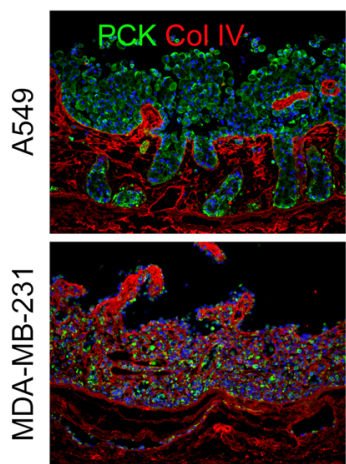

C

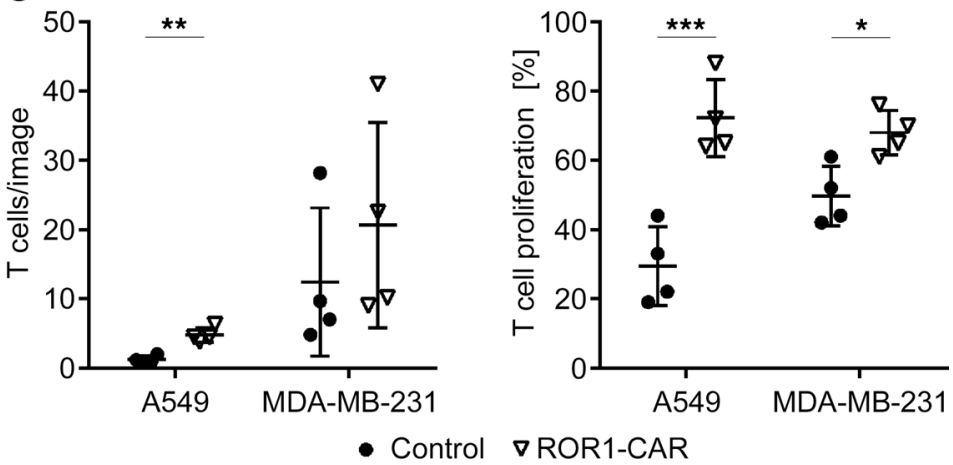

Figure 6. ROR1-CAR T cells migrate into tumor tissue and induce tumor cell lysis in a dynamic 3D culture. (A) Immunofluorescence staining of CD45 (green) on paraffin sections of dynamic tumor models treated with control or ROR1-CAR T cells. White arrows mark T cells that had migrated into the tissue matrix. Nuclei are counterstained with DAPI (blue). Scale bar: $100 \mu \mathrm{m}$. (B) Immunofluorescence double staining of PCK (green) and Col IV (red) on paraffin sections of untreated dynamic tumor models as well as tumor models treated with $\mathrm{CD}^{+}$and $\mathrm{CD} 8^{+}$untransduced control T cells or $\mathrm{CD} 4^{+}$ and CD8 ${ }^{+}$ROR1-CAR T cells with a total T cell number of $1 \times 10^{7}$ per condition. Scale bar: $100 \mu \mathrm{m}$. (C) Mean number of T cells per image and T cell proliferation assessed by quantification of Ki67/CD45 immunofluorescence double staining. $\mathrm{CD} 45^{+}$and $\mathrm{Ki} 67^{+} \mathrm{CD} 45^{+}$cells were counted in 10 images per condition. $n=4$. Data are presented as arithmetic mean $\pm \mathrm{SD}$, 2-tailed Student's $t$ test: ${ }^{*} P<0.05 ;{ }^{* *} P<$ $0.01 ;{ }^{* *} P<0.001$.

The data obtained in our 3D lung and breast cancer models show that ROR1-CAR T cells are able to penetrate and migrate through a tumor mass and confer a potent antitumor effect over a several-day period. In the static $3 \mathrm{D}$ model, the adherence and migration of $\mathrm{T}$ cells is supported by gravity. However, in the dynamic 3D model, ROR1-CAR $\mathrm{T}$ cells had to actively adhere to and migrate into the tumor from the constant medium flow. The bioreactor used for dynamic culture is constructed as a single circuit that provides an even amount of pressure throughout the system. The SISmuc matrix is connected to the circuit on both the apical and the basal sides, and therefore the same pressure applies on both sides. With these conditions, the bioreactor did not artificially favor ROR1-CAR $\mathrm{T}$ cell migration into the tumor mass. We have determined that the shear stress exerted on $\mathrm{T}$ cells in the bioreactor is $0.0004 \mathrm{dyn} / \mathrm{cm}^{2}$ (21), which is below the physiologic value measured in postcapillary venules of 3-30 $\mathrm{dyn} / \mathrm{cm}^{2}$ (26). By increasing the flow rate, shear stress can be enhanced to analyze its influence on $\mathrm{T}$ cell adherence and migration into the tumor tissue in future studies.

We provide evidence that ROR1-CAR $\mathrm{T}$ cells rapidly induced apoptosis of A549 and MDA-MB-231 tumor cells, produced high levels of cytokines, and were induced to productively proliferate in both the static and dynamic culture systems. ROR1-CAR T cells had a phenotype consistent with strong activation; i.e., they expressed high levels of CD25 and CD69. ROR1-CAR T cells were also induced to express PD-1, which is commonly observed in activated but also in exhausted T cells (27-29). Notably, we detected residual tumor cells at the end of the 3-day and 5-day time courses in both the static and dynamic culture systems, and with both the A549 lung cancer and the MDA-MB-231 breast cancer models. Our histopathologic analyses did not reveal whether these residual tumor cells had still been alive or whether they had been undergoing the process of apoptosis at the time of formalin fixation and paraffin embedding. However, we would speculate that these tumor cells had indeed been alive and had not been eliminated by ROR1-CAR T cells during the assay period. These data may indicate that the elimination of scattered residual tumor cells requires more time or that ROR1-CAR T cells were entering a state of exhaustion and were unable to eliminate these tumor cells. Accordingly, in future study protocols in our 3D tumor models, we will administer a second dose of ROR1-CAR T cells alone or administer a combination treatment of ROR1-CAR T cells and immune checkpoint inhibitors.

There is a continuous effort in the field to optimize existing and to develop novel CAR designs. We and others have shown in previous work that, for example, the choice of targeting domain, spacer design, and signaling module affect tumor cell recognition and antitumor function of CAR T cells ( 5 , 
11,30). However, there is presently no consensus on whether a single assay or a combination of in vitro and in vivo assays constitutes a "gold standard" for evaluating CARs and for predicting safety and efficacy in humans (18). It seems plausible, however, to assume that conventional 2D (in vitro) test systems may be more useful for assessing "short-term" CAR T cell effector functions, such as cytolytic activity and cytokine secretion, whereas animal models (in vivo) may provide a "long-term" readout of overall CAR T cell performance, including engraftment and proliferation. In this regard, the microphysiologic 3D models presented in this study are attractive because they provide information on both short- and long-term CAR $\mathrm{T}$ cell functions. We have performed several test campaigns with ROR1-CAR $\mathrm{T}$ cells in our 3D models and detected differences in antitumor function between CAR constructs with variable targeting domain, spacer domain, and costimulatory domain that we had also noted in 2D test systems and in murine xenograft models in previous work (5). Of particular interest, we supported superior antitumor functions with ROR1-CAR T cells expressing the "high-affinity" R12 targeting domain $(0.11 \mathrm{nM})$ compared with the "low-affinity" $2 \mathrm{~A} 2$ targeting domain $(0.42 \mathrm{nM})(31$, 32 ) in our 3D tumor models and supported the previously "optimized" R12 ROR1-CAR as the most potent construct, supporting its continuous preclinical and clinical development (9).

In the present study, we have focused on lung cancer and breast cancer to evaluate the antitumor function of ROR1-CAR T cells. The SISmuc 3D culture system can be readily adapted to accommodate other cancer entities, as we have recently demonstrated for colorectal cancer $(21,33)$. The $3 \mathrm{D}$ tumor models used in our study are based on a modular tissue engineering platform that can incorporate additional challenges for CAR T cells. In previous work, we have demonstrated the potential to generate vascularized tissue from our BioVaSc scaffold by engrafting the ECM matrix of the mesenteric artery and vein with human endothelial cells (34). Further, we have demonstrated the potential to include fibroblasts and other stroma cells into the extracellular SISmuc matrix and are currently developing protocols that allow inclusion of regulatory $\mathrm{T}$ cells and myeloid-derived suppressor cells into our $3 \mathrm{D}$ culture system to better simulate the immunosuppressive tumor microenvironment.

The BioVaSc was originally designed for the generation of artificial normal human tissues in regenerative medicine. Several normal human tissues, including trachea, myocardium, liver, skin, intestine, and kidney, have been generated on this platform $(12,35-40)$. Accordingly, we believe that there is a potential for assessing safety and tissue cross-reactivity of CAR T cells in 3D normal tissue models. With our existing protocols, it is possible to generate approximately 130 SISmuc scaffolds from 1 porcine jejunum. Each scaffold can then be populated with different types of normal or malignant cells. The microphysiologic 3D models described in our study may therefore contribute to reducing the number of animals in rodent and nonhuman primate models that are involved in preclinical CAR T cell research.

\section{Methods}

Tumor cell lines. The A549 (Leibnitz-Institut Deutsche Sammlung von Mikroorganismen und Zellkulturen [DSMZ], catalog ACC-107, Research Resource Identifier [RRID] CVCL_0023) and MDA-MB-231 (American Type Culture Collection [ATCC], catalog HTB-26, RRID CVCL_0062) cell lines were purchased from DSMZ and ATCC, respectively. Both cell lines were cultured in RPMI-1640 (Thermo Fisher Scientific) and $10 \%$ FCS (PAN-Biotech) as directed.

ROR1-CAR and preparation of CAR T cell lines. Lentiviral vectors encoding ROR1-CARs comprising a single-chain variable fragment derived from either the rabbit anti-human ROR1 mAb R12 or the mouse anti-human ROR1 mAb 2A2, a short IgG4-Fc hinge or long IgG4-Fc hinge- $\mathrm{C}_{\mathrm{H}} 2-\mathrm{C}_{\mathrm{H}} 3$ spacer, a CD28 trans-

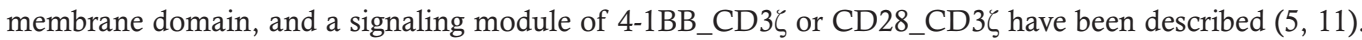
In each vector, the CAR transgene is separated by a T2A element from an EGFRt as transduction marker (41). $\mathrm{CD}^{+}$and $\mathrm{CD} 4^{+} \mathrm{T}$ cells were purified by immunomagnetic separation (Miltenyi Biotec) and activated with anti-CD3/CD28 beads (Thermo Fisher Scientific), before lentiviral transduction and enrichment and expansion of $\mathrm{CAR}^{+}$(i.e., EGFRt ${ }^{+}$) T cells (42).

SISmuc scaffold and preparation of lung and breast cancer models. The SISmuc scaffold of porcine SISmuc, and the preparation of 3D tissues under static and dynamic conditions, were performed according to established, standardized protocols $(12-14,43)$. In brief, jejunum was explanted and chemically decellularized with sodium deoxycholate monohydrate, and the mesentery and vascular tree were removed. After $\gamma$-sterilization, the SISmuc was fixed in a cell crown between 2 metal rings, and $1 \times 10^{5}$ A549 or MDA-MB-231 cells were seeded on the luminal side of the scaffold (Supplemental Figure 1A). The cell crowns were placed in 12-well plates 
with $2.5 \mathrm{ml}$ RPMI-1640 and 10\% FCS medium, and complete medium changes were performed every 2-3 days. After 11 days of culture, ROR1-CAR-modified or control T cells were administered directly into the medium. For ELISA analyses, medium was obtained from the cell crown. Before dynamic culture, tumor cells were allowed to attach to the SISmuc for 3 days under static conditions, and the scaffold was then placed into the cassette of a customized tubing system in a bioreactor (Supplemental Figure 1B and refs. 21 and 44). The tubing system contained $45 \mathrm{ml}$ of medium, and a roller pump (Cole-Parmer) continuously applied a constant medium flow of 3-4 ml/min. After 14 days of dynamic culture, ROR1-CAR-modified or control T cells were administered into a medium reservoir that was connected to the scaffold through an arterial and venous tube. For ELISA analyses, medium was aspirated through a sampling port.

ELISA for quantification of $M 30, I L-2$, and IFN- $\gamma$. ELISAs were performed according to the manufacturers' instructions (M30 Cytodeath ELISA, Peviva; IL-2 and IFN- $\gamma$ ELISA, BioLegend). All samples were analyzed as technical duplicates.

Reisolation of tumor cells. Tumor cells were reisolated from the SISmuc scaffold for subsequent flow cytometric analyses. The scaffolds were washed with PBS/EDTA and trypsin/EDTA applied for approximately 3 minutes, and then, the reaction was stopped with FCS. Cells were mobilized with a cell scraper (Sarstedt), passed through a $40-\mu \mathrm{m}$ cell sieve (Greiner Bio-One), pelleted by centrifugation for 5 minutes at $300 \mathrm{~g}$, and resuspended in flow buffer consisting of PBS (Thermo Fisher Scientific) with 0.5\% FCS (Thermo Fisher Scientific), 2 mM EDTA (Thermo Fisher Scientific), and $1.5 \mathrm{mM}$ sodium azide (AppliChem).

Phenotypic analysis by flow cytometry. Fluorochrome-conjugated mAbs against CD4 (Miltenyi Biotec, catalog 130-113-258, RRID AB_2726059), CD8 (Miltenyi Biotec, catalog 130-113-157, RRID AB_2725985), CD279 (PD-1, Miltenyi Biotec, catalog 130-117-384, RRID AB_2727929), ROR1 (Miltenyi Biotec, catalog 130-098-317, RRID AB_2653359), CD8a (BioLegend, catalog 301006, RRID AB_314124), CD69 (BioLegend, catalog 310906, RRID AB_314841) CD274 (PD-L1, BioLegend, catalog 329708, RRID AB_940360), and CD25 (BD Biosciences, catalog 561399, RRID AB_10643029) were used according to the manufacturers' instructions. The anti-EGFR mAb cetuximab (Bristol-Myers Squibb) was labeled in-house with Alexa Fluor 647 using the Alexa Fluor 647 Protein Labeling Kit (Thermo Fisher Scientific) following the manufacturer's instructions (Thermo Fisher Scientific). 7-AAD (BD) was used to exclude dead cells from analysis. An FACSCanto II (BD) was used for data acquisition, and data analyses were performed using FlowJo v10.0.7 software (Tree Star).

Immunofluorescence staining. Scaffolds were fixed in $4 \%$ paraformaldehyde and embedded in paraffin and $3-\mu \mathrm{m}$ cross sections prepared using a microtome (Leica Microsystems). Immunofluorescence staining was performed with primary antibodies against Col IV (Abcam, catalog ab6586, RRID AB_305584), vimentin (Abcam, catalog ab92547, RRID AB_10562134), Ki67 (Abcam, catalog ab16667, RRID AB_302459), CD45 (Agilent Dako, catalog M0701, RRID AB_2314143), and PCK (MilliporeSigma, catalog C2562, RRID AB_476839). All primary antibodies were diluted 1:100 and incubated overnight at $4^{\circ} \mathrm{C}$. Secondary antibodies against mouse IgG (Thermo Fisher Scientific, catalog A-31571, RRID AB_162542) conjugated to Alexa Fluor 647 or against rabbit IgG (Thermo Fisher Scientific, catalog A-31572, RRID AB_162543) conjugated to Alexa Fluor 555 were diluted 1:400 and incubated for 1 hour at room temperature. Nuclei were counterstained by DAPI (Southern-Biotech). Images were acquired using a digital microscope (BZ9000, Keyence) or a confocal laser scanning microscope (SP-8, Leica Microsystems) and stored electronically in TIFF format. Images were processed using the open-source software Fiji (https://fiji.sc). All images were processed using the identical algorithm.

Analysis of T cell proliferation by Ki67 staining. Immunofluorescence staining for CD45 and Ki67 was performed as described above. Six (dynamic conditions) or 10 (static) images per sample at original magnification $\times 20$ were analyzed, and the percentage of proliferating $\mathrm{T}^{-}$cells was determined by dividing the number of $\mathrm{CD} 45^{+} \mathrm{Ki} 67^{+}$by the number of $\mathrm{CD} 45^{+}$cells.

Statistics. The open-source software R (The Comprehensive R Archive Network) and GraphPad Prism 6.07 software (GraphPad Software) were used for statistical analyses. All experiments were independently repeated $n=4$ times unless stated otherwise. No samples were excluded from analysis. Two-tailed Student's $t$ test or Wilcoxon's rank-sum test were used with a confidence interval of $95 \%$, and results with a $P$ value of less than 0.05 were considered statistically significant.

Study approval. Peripheral blood was obtained from healthy donors after they provided written informed consent to participate in research protocols approved by the Institutional Review Board of the University of Würzburg (Ethik-Kommission der Universität Würzburg, Würzburg, Germany). 
The SISmuc scaffold was prepared from porcine intestine. All animals (local supplier) received humane care in compliance with the guidelines by the Federation of European Laboratory Animal Science Associations, WHO, and FDA (WHO-TRS978 Annex 3 and FDA-OCTGT Preclinical Guidance) after approval from the Institutional Animal Care and Use Committee of the University of Würzburg and the local competent authority (Regierung von Unterfranken, Würzburg, Germany).

\section{Author contributions}

SLN, GD, HW, and MH conceived the project and designed experiments. SLN, TS, GD, HW, and MH developed methodology. CG, LCN, LW, and JK acquired data. CG, LCN, LW, JK, TN, GD, SLN, and MH analyzed and interpreted data. CG, LCN, LW, JK, TN, HW, HE, GD, SLN, and MH wrote, reviewed, or revised the manuscript. TS provided administrative, technical, and material support. SLN, GD, HW, HE, and $\mathrm{MH}$ supervised the study.

\section{Acknowledgments}

This research was supported by German Cancer Aid (Deutsche Krebshilfe e.V.), Max Eder Program Grant no. 70110313 to $\mathrm{MH}$ ), Interdisziplinäres Zentrum für Klinische Forschung Würzburg (projects Z4/109 and D-244 to $\mathrm{MH}$ and project B-354 to $\mathrm{MH}$ and GD), and the BayernFIT program of the Bavarian state government (to HW and GD). MH is a member of the Young Scholar Program (Junges Kolleg) and Extraordinary Member of the Bavarian Academy of Sciences (Bayerische Akademie der Wissenschaften).

Address correspondence to: Michael Hudecek (for CAR T cells), Universitätsklinikum Würzburg, Medizinische Klinik und Poliklinik II, Oberdürrbacherstrasse 6, 97080 Würzburg, Germany. Phone: 4993120171092; Email: hudecek_m@ukw.de. Or to: Sarah L. Nietzer (for 3D models), Universitätsklinikum Würzburg, Tissue Engineering and Regenerative Medicine, Röntgenring 11, 97070 Würzburg, Germany. Phone: 499313182596; Email: sarah.nietzer@uni-wuerzburg.de.

1. Turtle $\mathrm{CJ}$, et al. CD19 CAR-T cells of defined $\mathrm{CD} 4^{+}: \mathrm{CD} 8^{+}$composition in adult B cell ALL patients. J Clin Invest. 2016;126(6):2123-2138.

2. Neelapu SS, et al. Axicabtagene ciloleucel CAR T-cell therapy in refractory large B-cell lymphoma. $N$ Engl J Med. 2017;377(26):2531-2544.

3. Maude SL, et al. Tisagenlecleucel in children and young adults with B-cell lymphoblastic leukemia. $N$ Engl J Med. 2018;378(5):439-448.

4. Hudecek M, et al. The B-cell tumor-associated antigen ROR1 can be targeted with T cells modified to express a ROR1-specific chimeric antigen receptor. Blood. 2010;116(22):4532-4541.

5. Hudecek M, et al. Receptor affinity and extracellular domain modifications affect tumor recognition by ROR1-specific chimeric antigen receptor T cells. Clin Cancer Res. 2013;19(12):3153-3164.

6. Baskar S, et al. Unique cell surface expression of receptor tyrosine kinase ROR1 in human B-cell chronic lymphocytic leukemia. Clin Cancer Res. 2008;14(2):396-404.

7. Zhang S, et al. The onco-embryonic antigen ROR1 is expressed by a variety of human cancers. Am J Pathol. 2012;181(6):1903-1910.

8. Balakrishnan A, et al. Analysis of ROR1 protein expression in human cancer and normal tissues. Clin Cancer Res. 2017;23(12):3061-3071.

9. Berger C, et al. Safety of targeting ROR1 in primates with chimeric antigen receptor-modified T cells. Cancer Immunol Res. 2015;3(2):206-216.

10. Yamaguchi T, et al. NKX2-1/TITF1/TTF-1-induced ROR1 is required to sustain EGFR survival signaling in lung adenocarcinoma. Cancer Cell. 2012;21(3):348-361.

11. Hudecek M, et al. The nonsignaling extracellular spacer domain of chimeric antigen receptors is decisive for in vivo antitumor activity. Cancer Immunol Res. 2015;3(2):125-135.

12. Linke K, Schanz J, Hansmann J, Walles T, Brunner H, Mertsching H. Engineered liver-like tissue on a capillarized matrix for applied research. Tissue Eng. 2007;13(11):2699-2707.

13. Stratmann AT, et al. Establishment of a human 3D lung cancer model based on a biological tissue matrix combined with a Boolean in silico model. Mol Oncol. 2014;8(2):351-365.

14. Göttlich C, et al. A combined 3D tissue engineered in vitro/in silico lung tumor model for predicting drug effectiveness in specific mutational backgrounds. J Vis Exp. 2016;(110):e53885.

15. Göttlich C, et al. A combined tissue-engineered/in silico signature tool patient stratification in lung cancer. Mol Oncol. 2018;12(8):1264-1285.

16. Sommermeyer $\mathrm{D}$, et al. Chimeric antigen receptor-modified $\mathrm{T}$ cells derived from defined $\mathrm{CD} 8^{+}$and $\mathrm{CD} 4^{+}$subsets confer superior antitumor reactivity in vivo. Leukemia. 2016;30(2):492-500.

17. Newick K, O'Brien S, Moon E, Albelda SM. CAR T cell therapy for solid tumors. Annu Rev Med. 2017;68:139-152.

18. Wegner A. Chimeric antigen receptor $\mathrm{T}$ cells for the treatment of cancer and the future of preclinical models for predicting their toxicities. Immunotherapy. 2017;9(8):669-680. 
19. Paszek MJ, et al. Tensional homeostasis and the malignant phenotype. Cancer Cell. 2005;8(3):241-254.

20. Walles T, Weimer M, Linke K, Michaelis J, Mertsching H. The potential of bioartificial tissues in oncology research and treatment. Onkologie. 2007;30(7):388-394.

21. Nietzer S, et al. Mimicking metastases including tumor stroma: a new technique to generate a three-dimensional colorectal cancer model based on a biological decellularized intestinal scaffold. Tissue Eng Part C Methods. 2016;22(7):621-635.

22. Kenny PA, et al. The morphologies of breast cancer cell lines in three-dimensional assays correlate with their profiles of gene expression. Mol Oncol. 2007;1(1):84-96.

23. Carterson AJ, et al. A549 lung epithelial cells grown as three-dimensional aggregates: alternative tissue culture model for Pseudomonas aeruginosa pathogenesis. Infect Immun. 2005;73(2):1129-1140.

24. McLachlan E, Shao Q, Wang HL, Langlois S, Laird DW. Connexins act as tumor suppressors in three-dimensional mammary cell organoids by regulating differentiation and angiogenesis. Cancer Res. 2006;66(20):9886-9894.

25. Pavesi A, et al. A 3D microfluidic model for preclinical evaluation of TCR-engineered T cells against solid tumors. JCI Insight. 2017;2(12):89762.

26. Valignat MP, Theodoly O, Gucciardi A, Hogg N, Lellouch AC. T lymphocytes orient against the direction of fluid flow during LFA-1-mediated migration. Biophys J. 2013;104(2):322-331.

27. John LB, et al. Anti-PD-1 antibody therapy potently enhances the eradication of established tumors by gene-modified T cells. Clin Cancer Res. 2013;19(20):5636-5646.

28. Barber DL, et al. Restoring function in exhausted CD8 T cells during chronic viral infection. Nature. 2006;439(7077):682-687.

29. Rupp LJ, et al. CRISPR/Cas9-mediated PD-1 disruption enhances anti-tumor efficacy of human chimeric antigen receptor T cells. Sci Rep. 2017;7(1):737.

30. Wallstabe L, Mades A, Frenz S, Einsele H, Rader C, Hudecek M. CAR T cells targeting $\alpha_{\mathrm{v}} \beta_{3}$ integrin are effective against advanced cancer in preclinical models. Adv Cell Gene Ther. 2018;1(2):e11.

31. Yang J, Baskar S, Kwong KY, Kennedy MG, Wiestner A, Rader C. Therapeutic potential and challenges of targeting receptor tyrosine kinase ROR1 with monoclonal antibodies in B-cell malignancies. PLoS One. 2011;6(6):e21018.

32. Baskar S, Wiestner A, Wilson WH, Pastan I, Rader C. Targeting malignant B cells with an immunotoxin against ROR1. MAbs. 2012;4(3):349-361.

33. Lübtow MM, et al. Drug induced micellization into ultra-high capacity and stable curcumin nanoformulations: physico-chemical characterization and evaluation in 2D and 3D in vitro models. J Control Release. 2019;303:162-180.

34. Mertsching $\mathrm{H}$, et al. Generation and transplantation of an autologous vascularized bioartificial human tissue. Transplantation. 2009;88(2):203-210.

35. Hoppensack A, et al. A human in vitro model that mimics the renal proximal tubule. Tissue Eng Part C Methods. 2014;20(7):599-609.

36. Steinke M, Dally I, Friedel G, Walles H, Walles T. Host-integration of a tissue-engineered airway patch: two-year follow-up in a single patient. Tissue Eng Part A. 2015;21(3-4):573-579.

37. Groeber F, et al. A first vascularized skin equivalent as an alternative to animal experimentation. ALTEX. 2016;33(4):415-422.

38. Schweinlin M, et al. Development of an advanced primary human in vitro model of the small intestine. Tissue Eng Part C Methods. 2016;22(9):873-883.

39. Weigel T, et al. A three-dimensional hybrid pacemaker electrode seamlessly integrates into engineered, functional human cardiac tissue in vitro. Sci Rep. 2018;8(1):14545.

40. Colunga T, et al. Human pluripotent stem cell-derived multipotent vascular progenitors of the mesothelium lineage have utility in tissue engineering and repair. Cell Rep. 2019;26(10):2566-2579.e10.

41. Wang X, et al. A transgene-encoded cell surface polypeptide for selection, in vivo tracking, and ablation of engineered cells. Blood. 2011;118(5):1255-1263.

42. Riddell SR, Greenberg PD. The use of anti-CD3 and anti-CD28 monoclonal antibodies to clone and expand human antigen-specific T cells. J Immunol Methods. 1990;128(2):189-201.

43. Schanz J, Pusch J, Hansmann J, Walles H. Vascularised human tissue models: a new approach for the refinement of biomedical research. J Biotechnol. 2010;148(1):56-63.

44. Schuerlein S, et al. A versatile modular bioreactor platform for tissue engineering. Biotechnol J. 2017;12(2):1600326. 